\title{
Why we need more psychology programmers/ EL Knife, a data utility for transforming spreadsheets
}

\author{
WALTER K. BEAGLEY \\ Alma College, Alma, Michigan
}

\begin{abstract}
The software needs of psychology researchersare sufficiently diverse that they cannot be met by the commercial sector. The field needs more psychologists who publish software, and individual researchers need to be able to write their own. To support this view, the author offers EL Knife, a data utility for reconfiguring multifactor spreadsheet files. Data may be divided by up to four factors, each of which may have an unlimited number of levels. The interface makes it easy to specify a wide variety of different configurations (different ways of packing multiple data dimensions into a two-dimensional spreadsheet). Many other possibilities for useful research software remain to be invented.
\end{abstract}

Software can be a curse. Like most people, I often feel guilty about all the software that sits idle in the box or on my hard drive because I have not yet had time to learn to use it. Even within the specialized area of psychology, there has been a lot of software development. Much of this has been described in Behavior Research Methods, Instruments, \& Computers or can be found in on-line software directories such as CTI Psychology (Hammond \& Trapp, 1991; www.psychology.ltsn.ac.uk/). So it comes as a surprise to me that I am going to argue that we need more software and more people writing it, especially more psychologists. The greatest need is software for research.

There are two reasons why we need more programmers. The first is to ensure the continued health and growth of psychology. Any research field that fails to improve and invent new tools will not be able to recruit the best young minds and will eventually dry up. The second reason has to do with giving individuals the greatest possible control of their own intellectual and professional lives. Researchers need to acquire at least journeyman-level programming skills so that their careers will not be limited by the bounds of somebody else's imagination. What I am suggesting is that we need both: more people who publish software for general use and more people who can write their own software. Let us consider some objections to this thesis.

Objection 1. Software creation should be left to wellfunded companies that can afford to develop and maintain quality products. It is true that a large company, em-

El Knife is available from the author by download (http://www.alma. edu/el). Individuals may download single copies for their own use for free. Institutions may contact the author to request a site license. All rights are reserved. The author has a financial interest in some of the software referred to in this paper. Correspondence concerning this article should be addressed to W. K. Beagley, Department of Psychology, Alma College, Alma, MI 48801 (e-mail: beagley @ alma.edu). ploying a full time team of software developers, can produce a more professional looking product than a lone psychology professor working over summer break. The problem is that software is expensive to develop and the market is too small (Schneider, 1991). Large software companies can find other, more lucrative opportunities for their money and talent. As a result, most psychology research software has been written by individuals and small companies with limited resources. This means that development for psychology is slower than in the commercial mainstream and lags behind changes in hardware and operating systems.

If large companies will not spend the money and small companies do not have it to spend, what can we do? Well, here is what we have done. First, we have relied on software that was written for a broader market. There is excellent software available for electronic instrumentation, digital imaging, and statistical analysis, because these functions are needed by scientists in all fields. Second, companies making software specifically for psychology research have survived by selling to the education market. Almost all of the research software packages for sale now have sets of experiments for use in undergraduate laboratory courses because, although still small, this market is much larger than that for pure research. This trend is good because it supports software that could not exist otherwise and it trains students on professional tools that they can continue to use as their research careers develop.

As with special purpose software in other fields, we have the problem of support and upgrade. Individual psychologists who write software may find it difficult to keep up with operating system changes or may find their interests changing. However, large commercial companies, having no commitment to psychology as such, are even more likely to cancel worthy projects if they do not prove sufficiently profitable. We all need to start thinking 
of software as being more like a service than a product. Those who want to sell software (or give it away) should understand that they need to make a long-term commitment to maintaining and improving the functionality that their users rely on.

Finally, the professional/amateur distinction cuts both ways. Most psychologists who write software are only amateur programmers. But is it really better to have software written by professional programmers who are, at best, only amateur psychologists? The bottom line is that we cannot rely totally on large software companies to provide the software for research. We need small companies and individuals with dual expertise.

Objection 2. Psychology researchers do not have time to learn and maintain a whole second set of professional skills, especially when the payoffs are so uncertain. This objection is hard to answer. Scientists who take time to write software to be used by others need to be rewarded for it. The two traditional currencies, money and professional publication credit, have not worked altogether well. Book publishers have paid people for software but have usually been interested in something cheap they can use as a lagniappe to help sell their real product, which is books. Psychologists who have marketed software on their own seldom report doing better than breaking even (Chute, 1993). And review committees for tenure and promotion often undervalue software development as a scholarly activity (Hammond \& Trapp, 1991). There may be a cognitive illusion operating here about which we need to educate our colleagues. Nonprogrammers appear to underestimate the complexity of software in much the same way that nonpsychologists underestimate the complexity of their own minds. In both cases, much of the complexity is hidden behind the smooth illusion of a good user interface. Just as our consciousness obscures the underlying mental and neural machinery, so the interface makes software appear much simpler than it really is.

With all this illusion, a piece of software that looks to be the equivalent of a short journal article may have involved more work than a major book. For example, the source code for my software package Eye Lines (Beagley, 1993) contains more than 40,000 lines of instructions. If printed out and bound into a book, it would be considerably longer than Herman Melville's Moby Dick. I don't claim any literary merit, but I faced difficulties unknown to Melville. He was writing in his native language; I was not. If he made a few misspellings, his book would still be fully functional; mine would not. And he did not have to revise his work every 3 or 4 years to keep it from becoming unreadable. So, one of the ways we could help programmers receive adequate credit is to educate nonprogrammers so that they have a better understanding and appreciation for the work.

Objection 3. Nonprofessionals will not produce useful software. This view is not unique to psychology. As the difficulty gap between using software and making soft- ware has appeared to widen, it has caused our entire education system to scale back the goals of computer literacy. In the late 1970 s and early 1980 s, all personal computers came bundled with a programming language, students were taught to write programs, and this produced the generation that is now building the Web. Today, few students are taught any programming. The prevailing wisdom is that most people do not like programming any better than they like math, and that spreadsheets and Web pages will prove more useful. These generalizations may be statistically true, but the same logic would lead us to shut down baseball leagues for children because most kids will never make the majors. If we want to continue to lead the world in developing whatever comes next, we need to give more kids a chance to find out whether they like programming.

Many of my colleagues nostalgically reminisce, "Remember when we used to write our own software?" Has it really gotten more difficult? Of course, it is difficult to compete with companies such as Microsoft, but it is still remarkably easy and fast to put together a program dedicated to running a specific experiment on a particular machine. And this is the power that researchers need. Ask yourself why we should require all psychology students to study statistics. The answers may apply equally well to programming. They are both technical, alien disciplines that increase a researcher's freedom to follow a problem wherever it leads, without being limited to off-the-shelf solutions.

Objection 4. Who needs software anymore anyway? Our future is out there on the Web where you can do or get anything you want or need, or if not yet, then soon. One of the reasons for the Web's popularity is that most Web sites are designed to require much less in the way of knowledge and planning on the part of the user than do most traditional software packages. Most of it, in fact, is just "Click here to go there." This is the ideal interface for information retrieval, but there is a limit to how much you can accomplish with this one spatial metaphor. So although the potentialities of the Web may be theoretically unlimited, those Web sites that perform more sophisticated functions will have to be more complex both to build and to use. We are going to need people who can mix HTML, Flash, Java, and other technologies to build the more complex Web sites that will help psychology researchers.

To sum up, I think that we need greater computer literacy, including some programming, in the population at large. In science, we should counsel students who want research careers to become at least moderately competent programmers. In academia, we need to build an environment that is supportive of those who want to go further and spend part of their careers in software development. Of course, we also need to push for better, easier to use software tools. We need all these things; our future depends on them. Over the past decade, members of SCiP have supported and advanced the field by introducing 
dozens of new programs at the annual meeting. In order to continue and encourage this tradition, I present the following offering.

\section{EL Knife: A Spreadsheet Utility for Reconfiguring Multidimensional Data Files}

The familiar spreadsheet stores data in two dimensions: rows and columns. To accommodate more complex designs, there are standard conventions for fitting multiple data dimensions into a single spreadsheet dimension. However, since there is always more than one way to configure such a data set, researchers sometimes have to reconfigure the data into a different format required for a particular analysis. This is necessary when data generated through the use of software are not correctly configured (Butler, 1988) or, more commonly, when a researcher needs to use two different statistical packages to perform different analyses. Changing the configuration is often done by manually reentering the data, by cut and paste editing, or by an ad hoc program that reads in the data, transforms it, and writes a new file (Wideman, 1991). All of these carry the risk of introducing errors. EL Knife automates the reconfiguration process. It loads data into a multidimensional matrix and presents the user with an interactive configuration map. This interface, originally developed for use with a proprietary format (Beagley, 1993), can be used to specify any possible spreadsheet configuration. Although existing statistical packages may allow some reconfiguration (e.g., Sall \& Lehman, 1996, p. 34), EL Knife supports a wider range of functions with a simpler interface. This gives a researcher the freedom to acquire or enter data in whatever form is most convenient, without worrying about how they are to be used.

Requirements. The Macintosh version of EL Knife requires Mac OS Version 7 or later. The PC version will run under DOS or Windows 3.1, 95, or 98. Either version will open files created on either platform. Data files should be in the form of tab-separated spreadsheets, created by spreadsheet, statistical, or word-processing software but saved as plain text, rather than in proprietary format. The data either can be numbers containing from 1 to 10 digits or can consist of single text characters.

Factors, levels, and the file header. Data can be divided by up to four factors, each of which can have an unlimited number of levels. For illustration, Figure 1 shows a small subset of data from an experiment on the effect of eye-scanning speed on the decrease in magnitude of the Müller-Lyer illusion (Ferriby \& Beagley, 1992). There are three experimental conditions, in which subjects either fixated on the center of the stimulus or scanned back and forth across the illusion figure at either a slow or a rapid (metronome-guided) pace. The change in illusion magnitude was measured by method of adjustment after 2 and 4 min of exposure. The 18 data points factor into 3 speeds $\times 3$ subjects $\times 2$ times.

When a file is opened, EL Knife looks for a header in the top line to identify what is contained in each column. Columns that contain labels for the various levels of a row

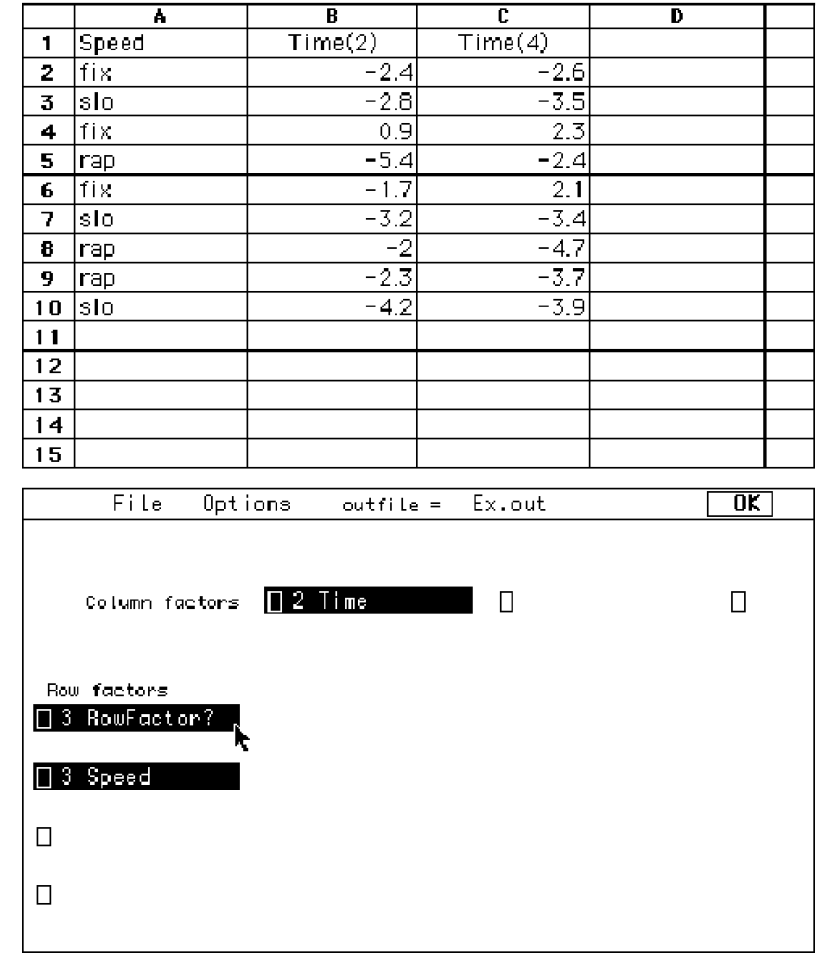

Figure 1. Sample data spreadsheet with configuration map. Column A is headed with the factor name "Speed" and contains labels for its three different levels. Columns $B$ and $C$ are headed with the factor name "Time," followed by one of its two level labels ( 2 or 4$)$. In the configuration map, speed is shown as a row factor because its levels appear on different rows of the spreadsheet. Time is shown as a column factor. Because each speed level appears three times, the map shows an additional row factor with three levels and a generic name. The data represent the change in illusion magnitude $(\mathrm{mm})$ produced by continuous exposure to the Müller-Lyer figure (see the text).

factor are headed with the name of that factor. Columns that contain the actual data are headed with factor names followed by level labels in parentheses. Level labels can include any legal character, but factor names should not include any numeric characters. The header at the top of Figure 1 specifies that speed is a row factor with labels in the first column and that time is a column factor with the data for Level 2 in column B and Level 4 in column C.

Configuration map, an interface for transforming the spreadsheet. After reading the file, the program presents a configuration map showing a rectangular block for each factor. Each block shows the factor name and number of levels. Its location on the map indicates whether it is a column factor or a row factor and its position in the hierarchical order. The map in Figure 1 has blocks for the speed and time factors. A third block labeled "RowFactor?" represents subjects that, in this example, were not labeled in the data file. Whenever EL Knife encounters repeated levels (or level combinations), it will create a new factor to differentiate them. The user can click on the block and change the generic name "RowFactor?" to 
something more meaningful, such as "Subject." This ability to detect unlabeled factors simplifies the preparation of data files. It is also useful for signaling errors, such as repeated subject codes or duplicated rows, either of which will produce an extra, unexpected factor block.

To reconfigure the spreadsheet, grab a block with the mouse and move it to a different location. You can take a column factor and change it into a row factor (or vice versa). Or you can rearrange the hierarchical order among multiple factors in either the row or the column dimension. To collapse a factor, click in the small box at the left end of the block to turn it off. The program will average together to single values all cells that are differentiated only by that factor. When you are satisfied, click on the OK button in the menu bar. The program will load the data into a virtual four-dimensional matrix, then write it back out to a new spreadsheet file with the chosen configuration.

The sample file in Figure 1 is arranged in the random order in which it was generated. Figure 2 shows how to sort by the speed factor to make the data easier to read. Use the mouse to grab the Speed block and drag it up to the first row factor position. This should bump Subject to the second row position. To write a revised file, click on the OK button in the menu bar. The user can view ei- ther the output file or the original input file by choosing one of the view commands under the File menu.

The resulting data configuration can be imported by most statistical packages and would allow running a two factor analysis of variance, with repeated measures for time. However, to graph these data, some statistical packages require that each condition be in a separate column. Drag the Speed block up to the column dimension. Place it either before or after the Time block, depending on which hierarchical order you prefer. The factor in the first position will change levels more slowly as you move across the columns. Click OK to produce the file shown in Figure 3.

Figure 4 demonstrates how to simplify the data file by collapsing the time factor. Click the mouse in the small box at the left end of the Time block. The program will average together the two Time cells for each subject, producing a spreadsheet that shows only the speed and subject factors. This ability to collapse factors also provides a quick way to look at means. For example, turning off the Subject block will give you the means of each of the six experimental conditions. Turning off both the Subject and the Time blocks will give you the means for the three speed groups.

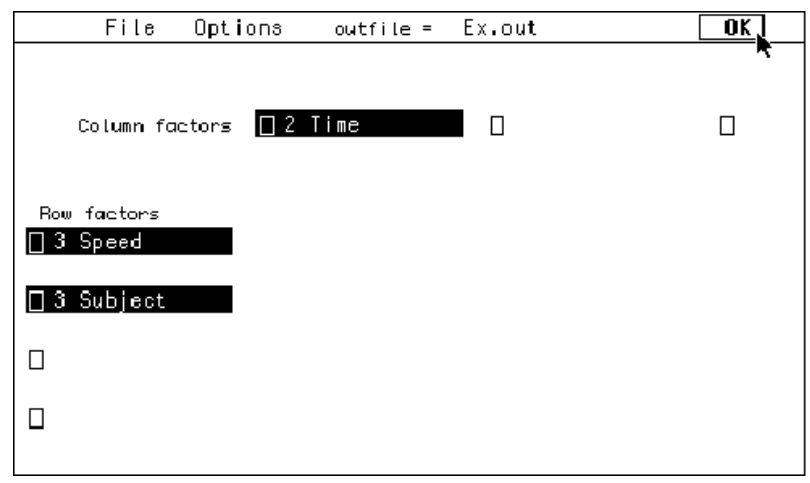

\begin{tabular}{|llll|}
\hline \multicolumn{2}{|l|}{ Click here or hit esc when done } & \multicolumn{1}{c|}{ Use cursor keys to scroll } \\
\hline Speed & Subject & Time(2) & Time(4) \\
fix & 1 & -2.4 & -2.6 \\
fix & 2 & .9 & 2.3 \\
fix & 3 & -1.7 & 2.1 \\
slo & 1 & -2.8 & -3.5 \\
slo & 2 & -3.2 & -3.4 \\
slo & 3 & -4.2 & -3.9 \\
rap & 1 & -5.4 & -2.4 \\
rap & 2 & -2 & -4.7 \\
rap & 3 & -2.3 & -3.7 \\
& & & \\
& & & \\
\end{tabular}

Figure 2. Sort by the speed factor. The positions of the Speed and the Subject blocks are interchanged on the map. In the resulting spreadsheet, the factor in first position (speed) changes levels most slowly as one moves down the rows.
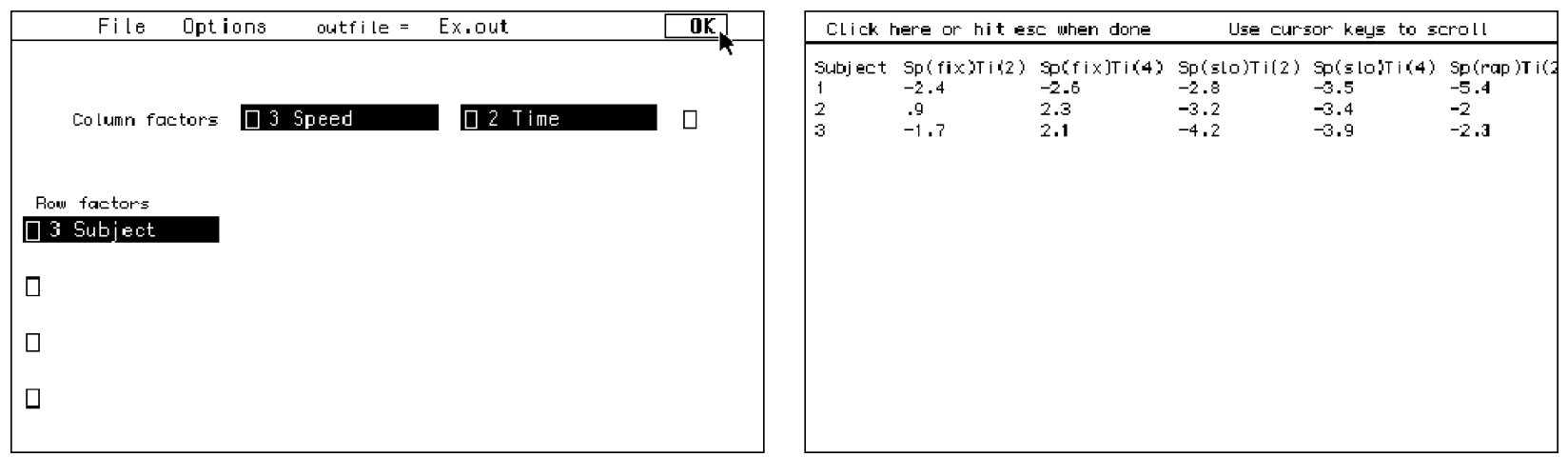

Figure 3. Move the speed factor from rows to columns. This places the data for each experimental condition in a separate column of the spreadsheet. Note the (optional) shortening of factor names in the column header when two (or more) factors are specified. The sixth column of data is off screen to the right, accessible though scrolling. 


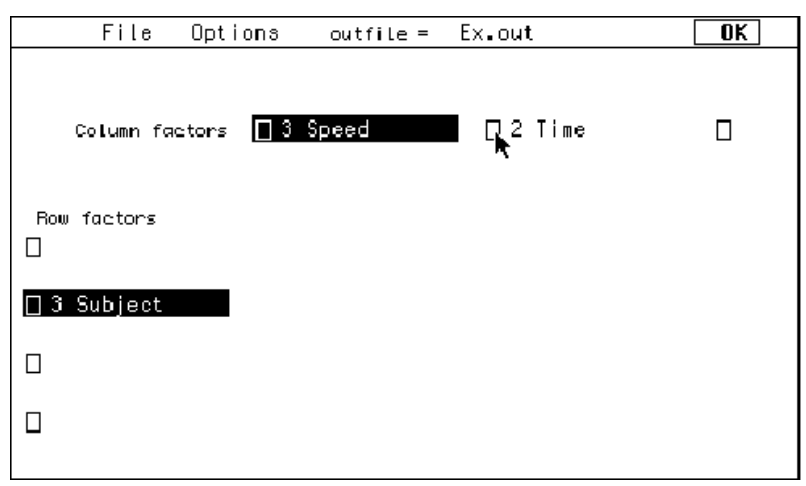

\begin{tabular}{|llll|}
\hline \multicolumn{3}{|c|}{ Cl ick here or hit esc when dane } & Use cur sor keys to scroll \\
\hline Subject & Speed(fix) & Speed(slo) & Speed(rap) \\
1 & -2.5 & -3.15 & -3.9 \\
2 & 1.6 & -3.3 & -3.35 \\
3 & .2 & -4.05 & -3 \\
& & & \\
& & & \\
& & \\
& & \\
\end{tabular}

Figure 4. Collapsing a factor. For each subject, the data for the two times are averaged together.

How it works. On opening a file, the program first checks for tab characters (ASCII character 9). If it finds them, it attempts to parse the top row as a header. If it finds parentheses, anything not in parentheses is a factor name, and anything inside parentheses is a level label. If there are no parentheses, it is assumed that alphabetic characters are factor names and that numerals specify level labels. Any column headed by just a factor name will be expected to contain the level labels for that factor. Any column headed with both factor name(s) and level label(s) will be expected to contain data. The program then reads through the rest of the file to acquire the labels for the various levels of each row factor. From this parsing, the program knows the number of factors and the number of levels of each. If the program detects two or more rows (or columns) with the same patterns of factor levels, it will create a new factor to differentiate them.

After the user has rearranged the factor blocks for the desired configuration and clicked on the OK button, the program assigns a number to each factor, starting with the first active row factor, proceeding through the active row factors followed by the active column factors, followed by any inactive (collapsed) factors. Then the file is opened a second time, and the data are read into a multidimensional array. They are written out to a new spreadsheet file by cycling through a nested loop structure that starts a new row for each unique combination of row factor levels, starts a new column for each unique combination of column level factors, and averages together the values in cells to be collapsed.

Other controls. An option menu allows the user to specify the number of decimal places. Those needing an output file that contains only numbers can choose to suppress the header and/or replace all level labels with numbers (denoting the order in which levels first appeared in the input file). In the file menu are choices that allow viewing either the input or the output spreadsheet.

Future. Possibilities for future versions of EL Knife include (1) a data editor that will be able to add or mod- ify headers and correct errors in labels or data, (2) import routines for data files that are not tab separated or legacy data files with no column separators, (3) alternate methods for importing data on the basis of different header formats or the use of hierarchical organization rather than headers, and (4) the ability to handle more than four dimensions.

My aim here has been to demonstrate that useful software can be produced without large development teams. SCiP can help by continuing to provide a forum for the exchange of ideas and by bringing together those who have needs and those who can build solutions. Countless possibilities for enhancing the future of psychology research await the creative invention of all our psychology programmers.

\section{REFERENCES}

BEAGLEY, W. K. (1993). Eye Lines: Generating data through image manipulation, issues in interface design, and the teaching of experimental thinking. Behavior Research Methods, Instruments, \& Computers, 25, 333-336.

ButLER, D. L. (1988). Selection of software in the instructional laboratory. Behavior Research Methods, Instruments, \& Computers, 20, 175-177.

Chute, D. L. (1993). MacLaboratory for Psychology: Successes, failures, economics, and outcomes over its decade of development. Behavior Research Methods, Instruments, \& Computers, 25, 180-188.

FerRIBY, J. A., \& BEAGLEY, W. K. (1992). Effects of scanning speed and scanning type on decrement of the Müller-Lyer illusion. Unpublished honors thesis, Alma College, Alma, Michigan.

Hammond, N., \& TrapP, A. (1991). Computers in psychology teaching in the U.K. Behavior Research Methods, Instruments, \& Computers, 23, 118-120. (www.psychology.ltsn.ac.uk/)

SALL, J., \& LehMAN, A. (1996). JMP start statistics. New York: Duxbury. SCHNEIDER, W. (1991). Equipment is cheap, but the field must develop and support common software for psychological research. Behavior Research Methods, Instruments, \& Computers, 23, 114-116.

WidEMAN, G. (1991). Streamlining experiment data manipulation with psychology experiment data interchange format (PXDIF). Behavior Research Methods, Instruments, \& Computers, 23, 288-291.

(Manuscript received November 24, 2000; accepted for publication March 21, 2001.) 